\title{
Selective involvement of ERK and JNK mitogen- activated protein kinases in early rheumatoid arthritis (1987 ACR criteria compared to 2010 ACR/EULAR criteria): a prospective study aimed at identification of diagnostic and prognostic biomarkers as well as therapeutic targets
}

\author{
Daphne de Launay, Marleen GH van de Sande, Maria JH de Hair, Aleksander M \\ Grabiec, Giijs PM van de Sande, K Aad Lehmann, Carla A Wijbrandts, \\ Lisa GM van Baarsen, Danielle M Gerlag, Paul P Tak, Kris A Reedquist
}

\begin{abstract}
- Additional data
(supplementary methods,

supplementary figures $1-4$ and supplementary tables $1-5$ ) are published online only. To view these files please visit the journal online (http://ard.bmj. com/content/71/3.toc)
\end{abstract}

Division of Clinical Immunology and Rheumatology, Academic Medical Center, University of Amsterdam, Amsterdam, The Netherlands

\section{Correspondence to}

Dr Kris A Reedquist, Division of Clinical Immunology and Rheumatology, Academic Medical Center, University of Amsterdam, Meibergdreef 9 , 1105 AZ, Amsterdam,

The Netherlands;

k.a.reedquist@amc.uva.nl

DdL and MGHvdS contributed equally to this manuscript. PPT and KAR contributed equally to this manuscript.

Received 20 September 2010 Accepted 14 August 2011 Published Online First 27 September 2011

\section{UNLOCKED}

This paper is freely available online under the BMJ Journals unlocked scheme, see http:// ard.bmj.com/info/unlocked.dtl

\begin{abstract}
Objectives To investigate the expression and activation of mitogen-activated protein kinases in patients with early arthritis who are disease-modifying antirheumatic drug (DMARD) naïve.

Methods A total of 50 patients with early arthritis who were DMARD naïve (disease duration $<1$ year) were prospectively followed and diagnosed at baseline and after 2 years for undifferentiated arthritis (UA), rheumatoid arthritis (RA) (1987 American College of Rheumatology (ACR) and 2010 ACR/European League Against Rheumatism (EULAR) criteria), or spondyloarthritis (SpA). Synovial biopsies obtained at baseline were examined for expression and phosphorylation of p38, extracellular signal regulated kinase (ERK) and c-Jun N-terminal kinase (JNK) by immunohistochemistry and digital analysis. Synovial tissue mRNA expression was measured by quantitative PCR (qPCR).
\end{abstract}

Results ERK and JNK activation was enhanced at inclusion in patients meeting RA criteria compared to other diagnoses. JNK activation was enhanced in patients diagnosed as having UA at baseline who eventually fulfilled 1987 ACR RA criteria compared to those who remained $U A$, and in patients with RA fulfilling 2010 ACR/EULAR criteria at baseline. ERK and JNK activation was enhanced in patients with RA developing progressive joint destruction. JNK activation in UA predicted 1987 ACR RA classification criteria fulfilment $\left(R^{2}=0.59, p=0.02\right)$ after follow-up, and disease progression in early arthritis $\left(R^{2}=0.16, p<0.05\right)$. Enhanced JNK activation in patients with persistent disease was associated with altered synovial expression of extracellular matrix components and CD44.

Conclusions JNK activation is elevated in RA before 1987 ACR RA classification criteria are met and predicts development of erosive disease in early arthritis, suggesting JNK may represent an attractive target in treating RA early in the disease process.

\section{INTRODUCTION}

Mitogen-activated protein kinase (MAPK) family members, namely p38 kinases $(\alpha-\delta)$, extracellular signal regulated kinases (ERKs) 1 and 2, and c-Jun $\mathrm{N}$-terminal kinase (JNKs) $1-3$, occupy critical positions in coupling diverse cell surface proteins, including antigen receptors, tumour necrosis factor (TNF) family receptors, chemokine and cytokine receptors, and Toll-like receptors to inflammatory gene expression. ${ }^{12}$ Members of each MAPK family are expressed and activated in synovial tissue of patients with rheumatoid arthritis (RA) and other forms of inflammatory arthritis. ${ }^{3-5}$ Highly selective pharmacological inhibitors of p38, ${ }^{6-9} \mathrm{ERK}^{10} 11$ and JNK ${ }^{31213}$ prevent inflammatory activation of stromal fibroblast-like synoviocytes (FLS) derived from synovial tissue, chondrocytes and osteoclasts from patients with RA. Additionally, pharmacological inhibition or genetic deletion of MAPK activity reduces inflammation and joint destruction in multiple experimental animal models of RA. ${ }^{68101214-18}$ These data collectively suggest that therapeutic strategies inhibiting MAPK activation may be useful in the treatment of RA. ${ }^{12} 1920$

Despite this wealth of preclinical analyses, little is known about the distinct contributions of each MAPK to the onset and perpetuation of RA. Clinical parameters and biomarkers have yet to be identified which are associated with synovial MAPK activation status, and MAPK activation in RA has primarily been examined in patients with destructive end-stage disease..$^{3-5}$ In the transgenic human TNF model of murine arthritis, p38 activation is required for induction of inflammation and joint destruction. ${ }^{15} 21$ Whether this observation can be translated into successful treatment of RA with MAPK inhibitors, especially in early disease, is uncertain however, as clinical trials with p38 inhibitors have not been successful. ${ }^{22}{ }^{23}$ Recent kinetic analyses of MAPK activation in experimental murine arthritis have revealed model-specific differences in the degree of p38, ERK and JNK activation, as well as in the timing of their activation during disease onset and resolution. ${ }^{24}$ Here, we examined if similar differences in MAPK involvement might be relevant to the earliest stages of the development of RA, by 
assessing the relationship between MAPK expression and activation, and disease diagnosis and outcome in a prospective cohort of patients with early arthritis who were disease-modifying antirheumatic drug (DMARD) naïve.

\section{PATIENTS AND METHODS}

\section{Patients}

A total of 50 patients with arthritis of duration of less than 1 year, as measured from the first clinical signs of arthritis, irrespective of which joint was initially affected, and a clinically inflamed knee, ankle or wrist joint, underwent arthroscopic synovial biopsy. Diagnosis of RA or spondyloarthritis (SpA) was made according to established classification criteria. ${ }^{25-28}$ Patients were classified as having undifferentiated arthritis (UA) if no classifying diagnosis for RA, SpA or other forms of arthritis could be made. After 2 years of follow-up final diagnosis was made according to classification criteria. All patients were naïve to treatment with DMARDs and corticosteroids at inclusion, and after baseline study procedures all patients were treated consistent with European League Against Rheumatism (EULAR) guidelines. ${ }^{29}$ In case of a clinical diagnosis of RA, DMARD treatment was initiated directly after baseline study procedures were completed. The 28-joint Disease Activity Score (DAS28) was systematically determined and patients were treated according to the treat-to-target principle, aiming for DAS28<2.6, using conventional DMARDs, corticosteroids and biologicals, if indicated. If a combination of DMARDs did not result in a DAS28 $<3.2$ then a biological was given. Upon decision of the treating doctor corticosteroids were given in combination with a DMARD, either high dose and tapered down in 6-8 weeks or more prolonged low dose. In case of a diagnosis of $\mathrm{SpA}$ with peripheral arthritis, all patients, except patients with reactive arthritis were started on methotrexate. The patients with reactive arthritis were treated with intra-articular steroids and non-steroidal anti-inflammatory drugs. Treatment was aimed at minimal disease activity. Patients with UA were treated with intra-articular steroids and, if arthritis was persistent, a DMARD was given.

At inclusion we assessed disease activity (68 tender and 66 swollen joint score, patient's visual analogue scale (VAS) of global disease activity (scale 0-100 mm), VAS of pain (scale 0-100 mm), erythrocyte sedimentation rate (ESR) and C-reactive protein (CRP) levels) and collected serum and arthroscopic synovial tissue biopsy samples. X-rays were obtained at baseline and after 2 years; erosion scoring was based on the presence or absence of erosions on $\mathrm{x}$-rays of hands and feet where the modified Sharpvan der Heijde erosion score was $\geq 1$. $^{30}$ This study was approved by the institutional review board, performed according to the declaration of Helsinki. All study patients provided written informed consent.

\section{Measurement of serum autoantibodies}

Patient serum immunoglobulin $M$ rheumatoid factor (IgM-RF) and anti-citrullinated protein antibodies (ACPA) were measured using IgM-RF (Sanquin, Amsterdam, The Netherlands) and anticyclic citrullinated peptide 2 (CCP2) (Eurodiagnostica, Arnhem, The Netherlands) ELISA kits.

\section{Synovial tissue biopsy sampling, immunohistochemistry and gene expression}

All patients underwent arthroscopic synovial tissue biopsy sampling of an actively inflamed ankle, wrist or knee joint. ${ }^{31}$ Synovial tissue biopsies were collected from each patient, stored and processed for immunohistochemistry, and stained sections analysed by digital imaging analysis as previously described. ${ }^{32-34}$ Detailed descriptions of stainings, analyses and mRNA expression studies are provided in the supplementary methods.

\section{Statistics}

Statistical analysis was performed using Windows GraphPad Prism 4 (GraphPad Software, La Jolla, California, USA) and SPSS V.16.0 (SPSS, Chicago, Illinois, USA) software. Comparisons in expression or phosphorylation of markers between cohorts were performed using the Mann-Whitney U test, first using the Kruskal-Wallis test when more than two groups were compared. Logistic regression analysis was used to analyse the relationship between relative MAPK phosphorylation and development of RA and the development of erosive disease. Results were considered statistically significant if $\mathrm{p}<0.05$.

\section{RESULTS}

\section{p38, ERK and JNK are differentially expressed and phosphorylated in patients with early arthritis with distinct diagnoses}

We performed immunohistochemical staining on synovial biopsy samples using antibodies recognising total and p-p38, ERK and JNK MAPKs. Within the cohort, 27 patients were diagnosed as having RA at 2 years after enrolment in the study based on 1987 American College of Rheumatology (ACR) criteria for RA, 7 with SpA and 16 with UA. Clinical characteristics of each patient group are shown in table 1. p38 expression was highest in UA, and significantly different in this group compared to $\mathrm{SpA}(\mathrm{p}<0.05)$, but not RA (supplementary figure 1). The p-p38 levels were higher in UA than SpA $(p<0.05)$. ERK expression was significantly higher in RA than SpA $(p<0.01)$ and UA $(p<0.01)$ (supplementary figure 1). $p$-ERK levels were also highest in RA, where it was elevated compared to SpA $(p<0.01)$ and UA $(p<0.01)$. No differences in JNK expression were noted between diagnostic groups (supplementary figure $1)$, but $p-J N K$ levels were higher in RA than in SpA $(p<0.005)$ and UA $(p<0.01)$. Within each diagnostic group, and across the cohort as a whole, no significant correlation was observed between MAPK expression and phosphorylation levels, suggesting that phosphorylation was driven by differential inflammatory stimuli input (data not shown, see supplementary figure 2 for representative photomicrographs of staining distribution in each diagnostic group).

\section{Relative phosphorylation of ERK and JNK is enhanced in patients with early RA}

To gain insight into the degree of MAPK engagement by inflammatory stimuli, we calculated the relative phosphorylation of p38, ERK and JNK proteins for each patient (IOD phosphoMAPK/IOD total MAPK, arbitrary units). Relative p38 phosphorylation was similar in all diagnostic groups (figure $1 \mathrm{~A}$, left panel). In contrast, relative ERK phosphorylation in RA was higher than in SpA $(p<0.05)$ and UA $(p<0.01)$ (figure 1A, middle panel). Relative JNK phosphorylation (figure $1 \mathrm{~A}$, right panel) was elevated in RA compared to UA $(\mathrm{p}<0.01)$ and SpA $(\mathrm{p}<0.005)$. Using 2010 ACR/EULAR classification criteria, 35 patients were diagnosed as having RA, 7 with SpA and 8 with UA at baseline (supplementary table 1). Here, no differences in relative p38 or ERK phosphorylation were observed, but relative JNK phosphorylation was elevated in RA $(\mathrm{p}<0.001)$ compared to $\mathrm{SpA}$ (figure 1B). 
Table 1 Characteristics of study patients

\begin{tabular}{lccll}
\hline & UA $>>\mathbf{U A}, \mathbf{n = 1 6}$ & $\mathbf{R A}, \mathbf{n = 1 9}$ & UA $>>\mathbf{R A}, \mathbf{n = 8}$ & SpA, $\mathbf{n = 7}$ \\
\hline Age, years & $39(20-67)$ & $53(22-82)$ & $54(43-66)$ & $44(20-56)$ \\
Female, $\mathrm{n}$ & 12 & 10 & 7 & 0 \\
Disease duration, months & $4(1-10)$ & $5(1-12)$ & $4(1-11)$ & $2(1-12)$ \\
VAS, $0-100$ & $63(9-93)$ & $37(11-99)$ & $48(32-98)$ & $35(17-91)$ \\
TJC68, $\mathrm{n}$ & $3(0-37)$ & $16(5-38)$ & $13(3-25)$ & $4(0-11)$ \\
SJC66, $\mathrm{n}$ & $1(1-19)$ & $8(4-41)$ & $6(1-20)$ & $2(1-5)$ \\
ESR, mm/h & $34(4-85)$ & $32(3-91)$ & $30(14-77)$ & $11(3-47)$ \\
CRP, mg/litre & $11(2-58)$ & $12(3-133)$ & $173-42$ & $7(1-46)$ \\
IgM-RF positive, $n$ & 2 & 13 & 1 & 0 \\
ACPA positive, $n$ & 2 & 10 & 3 & 0 \\
No DMARDs & 12 & 0 & 0 & 2 \\
DMARD monotherapy & 3 & 15 & 4 & 5 \\
$\geq 2$ DMARDs & 1 & 4 & 2 & 0 \\
$\geq 2$ MTX plus anti-TNF & 0 & 0 & 2 & 0 \\
\hline
\end{tabular}

Patients were classified as having rheumatoid arthritis (RA) based on 1987 American College of Rheumatology (ACR) criteria. UA $>>U A=$ undifferentiated arthritis (UA) at baseline and at 2 years of follow-up; UA $>>R A=$ classified as having UA at baseline and diagnosed as having RA at 2 year follow-up.

ACPA, anti-citrullinated protein antibody; CRP, C-reactive protein; DMARD, disease-modifying antirheumatic drug; ESR, erythrocyte sedimentation rate; IgM-RF, immunoglobulin M rheumatoid factor; MTX, methotrexate; SpA, spondyloarthritis; SJC66, 66-joint swollen joint count; TJC68, 68-joint tender joint count; TNF, tumour necrosis factor $\alpha$; VAS, visual analogue scale of global disease activity.

A

\section{7 criteria}

p38

ERK

JNK
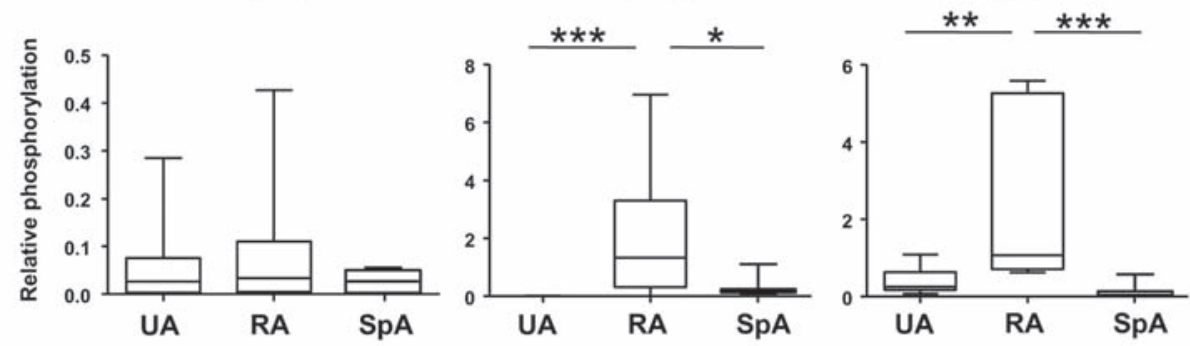

B

\section{0 criteria}

p38

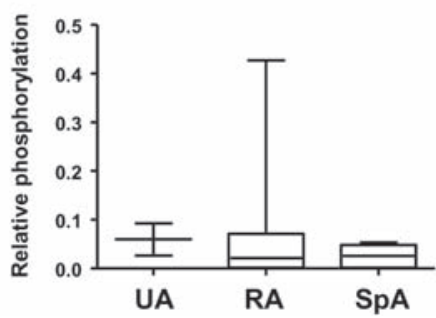

ERK

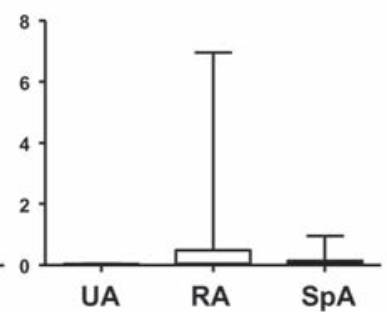

JNK

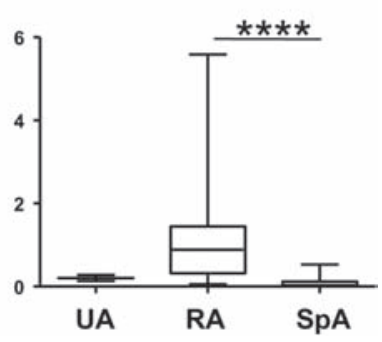

Figure 1 Relative mitogen-activated protein kinase (MAPK) activation in relation to disease diagnosis. Quantitative comparison of relative MAPK phosphorylation in the synovial tissue of patients with early arthritis. Tissue sections from patients diagnosed as having rheumatoid arthritis (RA) according to 1987 American College of Rheumatology (ACR) criteria, spondyloarthritis (SpA) and undifferentiated arthritis (UA) after 2 years of follow-up were stained with antibodies against phospho- and total p38, extracellular-signal regulated kinase (ERK) and c-Jun N-terminal kinase (JNK). Stainings were developed with biotin tyramide enhancement, horseradish peroxidase and aminoethylcarbazole, followed by counterstaining with Mayer's haematoxylin, and evaluated by digital imaging analysis. Relative phosphorylation levels (ratio of IOD phosphorylated protein to IOD of total protein, arbitrary units) of p38, ERK and JNK were calculated for patients diagnosed as having RA according to (A) 1987 ACR criteria and (B) 2010 ACR/European League Against Rheumatism (EULAR) criteria, SpA and UA. Data are presented as boxplots, where the boxes represent the 25th to 75th percentiles, the lines within the box mark the median value, and lines outside the boxes denote the 10th and 90th percentiles. Lines connecting data sets indicate statistically significant differences between groups. ${ }^{*} p<0.05 ;{ }^{* *} p<0.01$; ${ }^{* *} p<0.005$. 
A

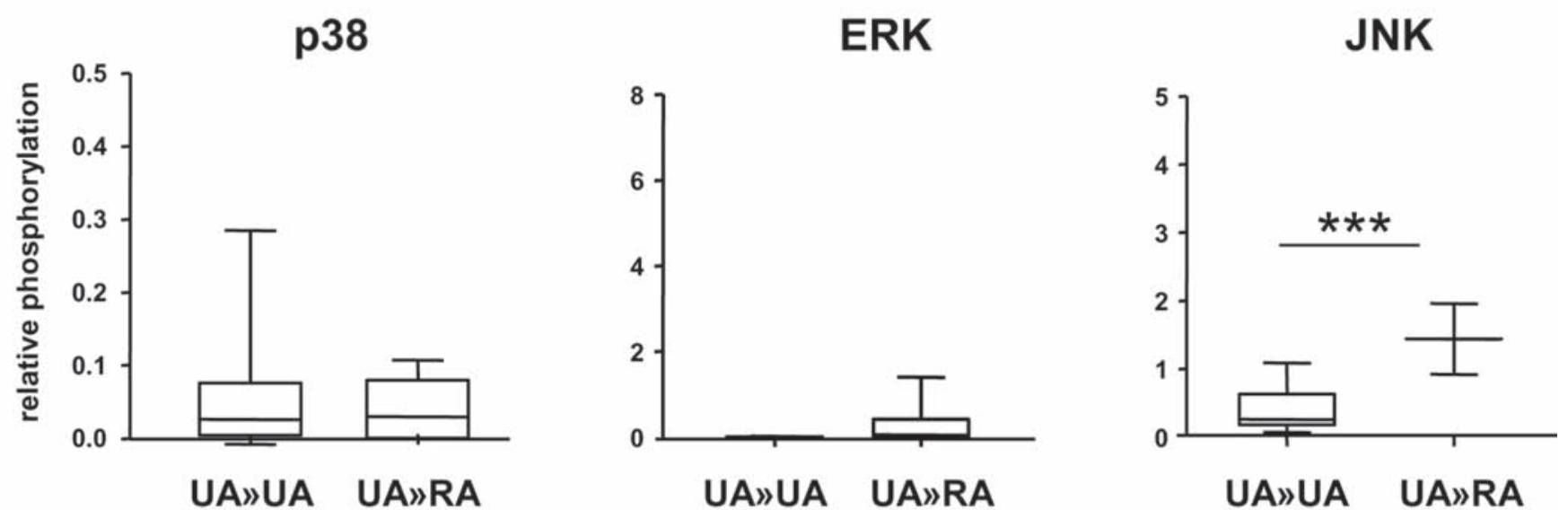

B

\section{RA patients (1987 criteria)}
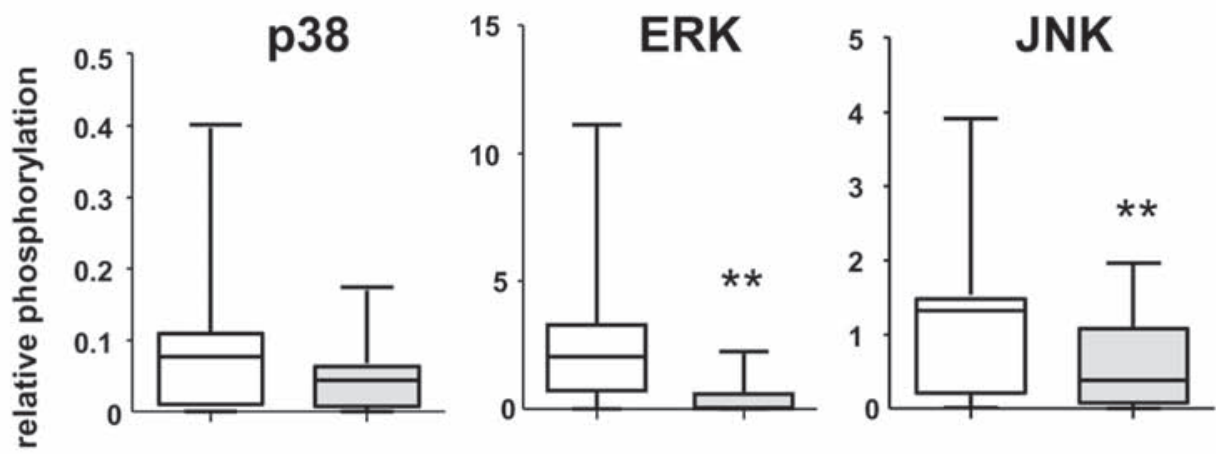

\section{Early arthritis patients}
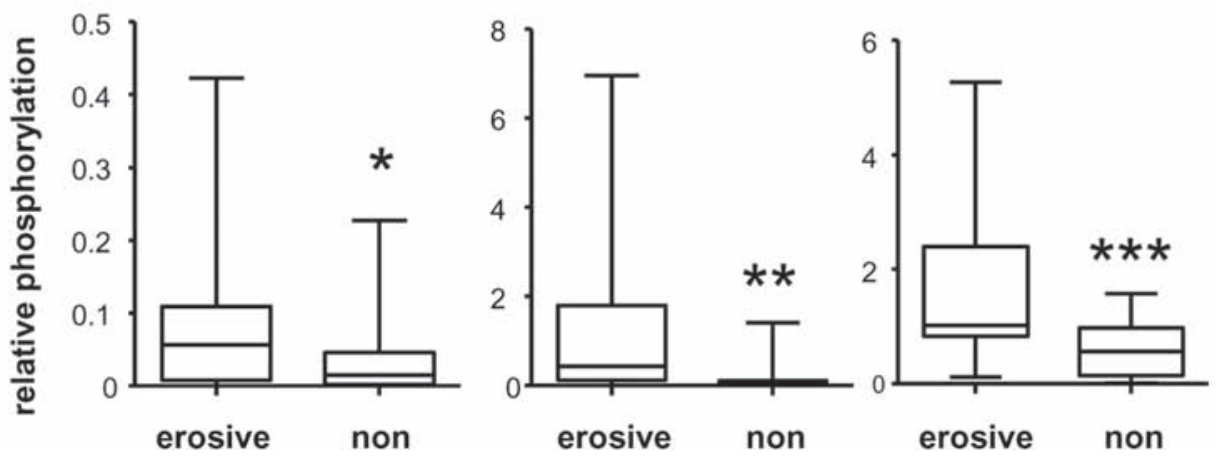

Figure 2 Relative mitogen-activated protein kinase (MAPK) activation in relation to disease outcome and progression. A. Quantitative comparison of relative MAPK phosphorylation in the synovial tissue of patients classified according to 1987 American College of Rheumatology (ACR) criteria as undifferentiated arthritis (UA) which remained UA after 2 years (UA $>>U A$ ), and UA which was diagnosed as rheumatoid arthritis (RA) after 2 years (UA $>>$ RA). Relative phosphorylation levels of $p 38$, extracellular signal regulated kinase (ERK) and c-Jun N-terminal kinase (JNK) were calculated for patients diagnosed as having UA $>>U A$ and $U A>>R A$. B. Quantitative comparison of relative MAPK phosphorylation in the synovial tissue of patients classified as having RA after 2 years based on 1987 ACR criteria (upper panels) and all patients with early arthritis with persistent erosive and persistent non-erosive disease (lower panels).

\section{Relative phosphorylation of ERK and JNK is enhanced in patients with early RA before fulfilment of 1987 ACR RA classification criteria}

We next compared MAPK activation with the presence of RF and ACPA, autoantibodies predictive of the development, disease course and prognosis of RA. ${ }^{35} 36$ Patients with RA and UA receiving a diagnosis of $R A$ at 2 year follow-up (UA $>$ RA) identified by 1987 ACR criteria were grouped based on the absence or presence of serum RF or ACPA at baseline (see supplementary tables 2 and 3 for patient clinical characteristics). Relative activation of ERK $(p<0.0005)$ and JNK $(p<0.005)$ was significantly higher in patients who were RF+ $(n=14)$ than RF- $(n=13)$. Patients who were ACPA+ $(n=13)$ also displayed enhanced ERK $(p<0.001)$ and JNK $(p<0.005)$ activation compared to patients who were ACPA- $(n=14)$ (supplementary figure 3).

Given that ERK and JNK activation was elevated in patients who were RF and ACPA seropositive, we next examined if 


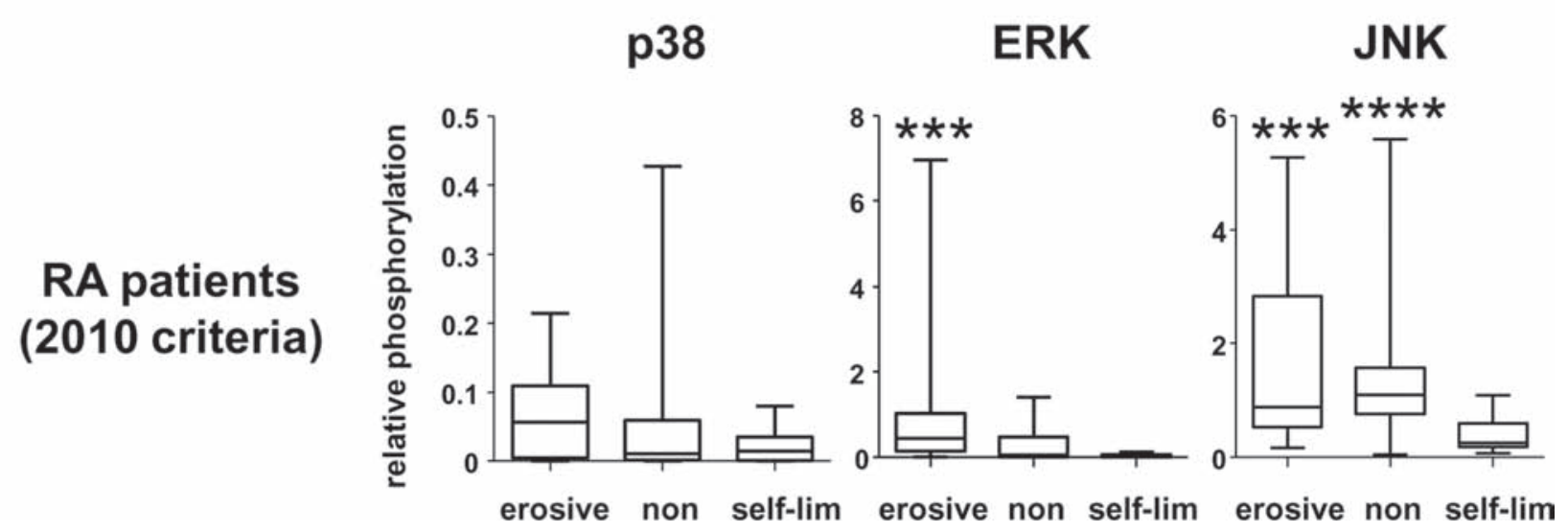

Figure 2 (Continued) C. Comparison of relative MAPK phosphorylation in the synovial tissue of patients classified with RA according to 2010 ACR/EULAR criteria displaying self-limiting, persistent non-erosive and erosive disease. Erosive disease was defined by a Sharp-van der Heijde erosion score $\geq 1$ at 2 year follow-up. Relative MAPK phosphorylation was calculated as the ratio of IOD phosphorylated protein to IOD of total protein (arbitrary units). Data are presented as boxplots, where the boxes represent the 25th to 75th percentiles, the lines within the box mark the median value, and lines outside the boxes denote the 10th and 90th percentiles. Lines connecting data sets indicate statistically significant differences between groups. ${ }^{*} \mathrm{p}<0.05 ;{ }^{*} \mathrm{p}<0.01 ;{ }^{* * *} \mathrm{p}<0.005$.

ERK and JNK might already be activated in UA $>>$ RA, as diagnosed by 1987 ACR criteria (see table 1 for patient characteristics). We observed no differences in relative p38 (figure 2A, left panel) or ERK (figure $2 \mathrm{~A}$, middle panel) phosphorylation between patients diagnosed as having UA who remained UA (UA $>>U A)(n=16)$ and patients who progressed from $U A>R A(n=8)$. In contrast, JNK phosphorylation was elevated in $U A>>R A$ (figure $2 \mathrm{~A}$, right panel) compared to $U A>>U A$ $(p<0.005)$. Univariate logistic regression analysis showed that relative JNK activation at baseline was significantly related to fulfilment of classification criteria for RA after follow-up with an explained variance of $59 \%\left(R^{2}=0.59, p=0.02\right)$. Thus, in patients with early arthritis, elevated ERK and JNK activity distinguish RA from other forms of arthritis, and JNK activation is elevated in patients with RA even before 1987 ACR classification criteria of RA are met. Similar regression analyses based on 2010 ACR/EULAR classification criteria could not be performed due to the small number $(n=2)$ of patients with $\mathrm{UA}>>\mathrm{UA}$ (supplementary figure 4).

\section{Relative ERK and JNK activation is elevated in patients with RA developing erosive disease}

To examine the relationship between MAPK activation and disease outcome in patients with RA, RA $>>R A$ and UA $>$ RA were pooled and grouped based on the development of erosive disease (defined as a modified Sharp-van der Heijde erosion score was $\geq 1$ at 2 year follow-up, see table 2 for patient clinical characteristics). ${ }^{30}$ Only one patient with early arthritis had erosive disease at baseline. Relative p38 phosphorylation was similar between patients with RA with non-erosive $(n=15)$ and erosive disease $(n=12)$ (figure $2 B$, upper left panel). Relative ERK $(\mathrm{p}<0.01)$ (figure $2 \mathrm{~B}$, upper middle panel) and JNK phosphorylation $(p<0.01$ ) (figure $2 B$, upper right panel) was higher in patients developing erosive disease. Examining all patients with early arthritis, regardless of diagnosis (see table 3 for patient characteristics), activation of p38 ( $<<0.05)$, ERK $(p<0.005)$ and JNK $(p<0.001)$ was elevated in patients with erosive disease (figure $2 \mathrm{~B}$, lower panels). JNK activation, but not p38 or ERK activation, predicted development of erosive disease $\left(R^{2}=0.16, p<0.05\right)$.

Table 2 Characteristics of patients with rheumatoid arthritis (RA) with erosive and non-erosive disease

\begin{tabular}{lcll}
\hline & RA erosive, $\mathbf{n = 1 2}$ & RA non-erosive, $\mathbf{n = 1 5}$ & p Value \\
\hline Age, years & $53(24-82)$ & $54(22-58)$ & 0.88 \\
Disease duration, months & $5(1-10)$ & $4(1-12)$ & 0.86 \\
DAS28 & $5.0(2.9-6.6)$ & $5.9(3.3-6.7)$ & 0.13 \\
ESR, mm/h & $37(3-91)$ & $27(7-76)$ & 0.67 \\
CRP, mg/litre & $9(3-114)$ & $16(3-133)$ & 0.62 \\
VAS, 0-100 & $27(11-76)$ & $50(25-98)$ & 0.12 \\
IgM-RF positive, $\%$ & 66 & 43 & 0.27 \\
ACPA positive, $\%$ & 44 & 50 & 0.80 \\
DMARD monotherapy & 9 & 8 & \\
$\geq 2$ DMARDS & 3 & 5 & \\
MTX plus anti-TNF $\alpha$ & 0 & 2 &
\end{tabular}

Patients were classified as having rheumatoid arthritis (RA) based on 1987 American College of Rheumatology (ACR) criteria.

ACPA, anti-citrullinated protein antibody; CRP, C-reactive protein; DAS28, 28-joint Disease Activity Score; DMARD, disease-modifying antirheumatic drug; ESR, erythrocyte sedimentation rate; IgM-RF, immunoglobulin M rheumatoid factor; MTX, methotrexate; TNF, tumour necrosis factor; VAS, visual analogue scale of global disease activity. 
A

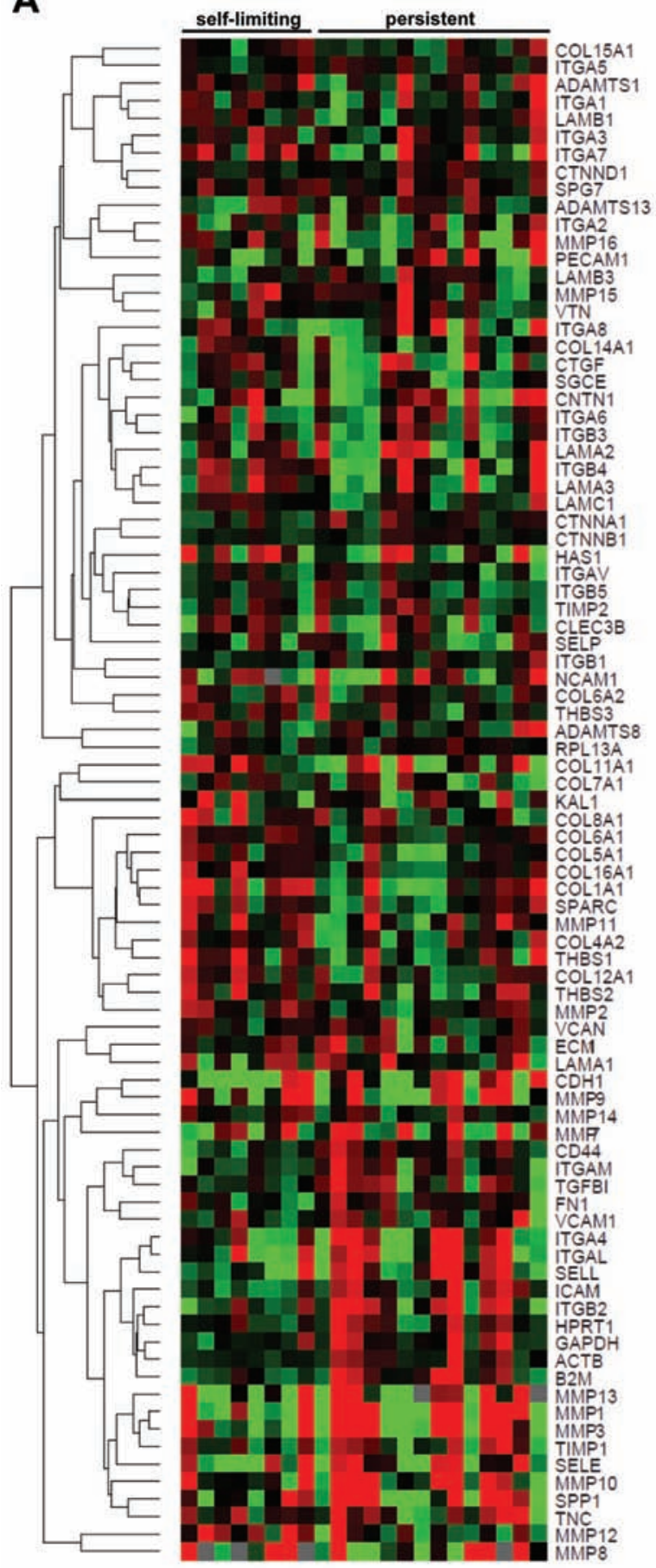

B
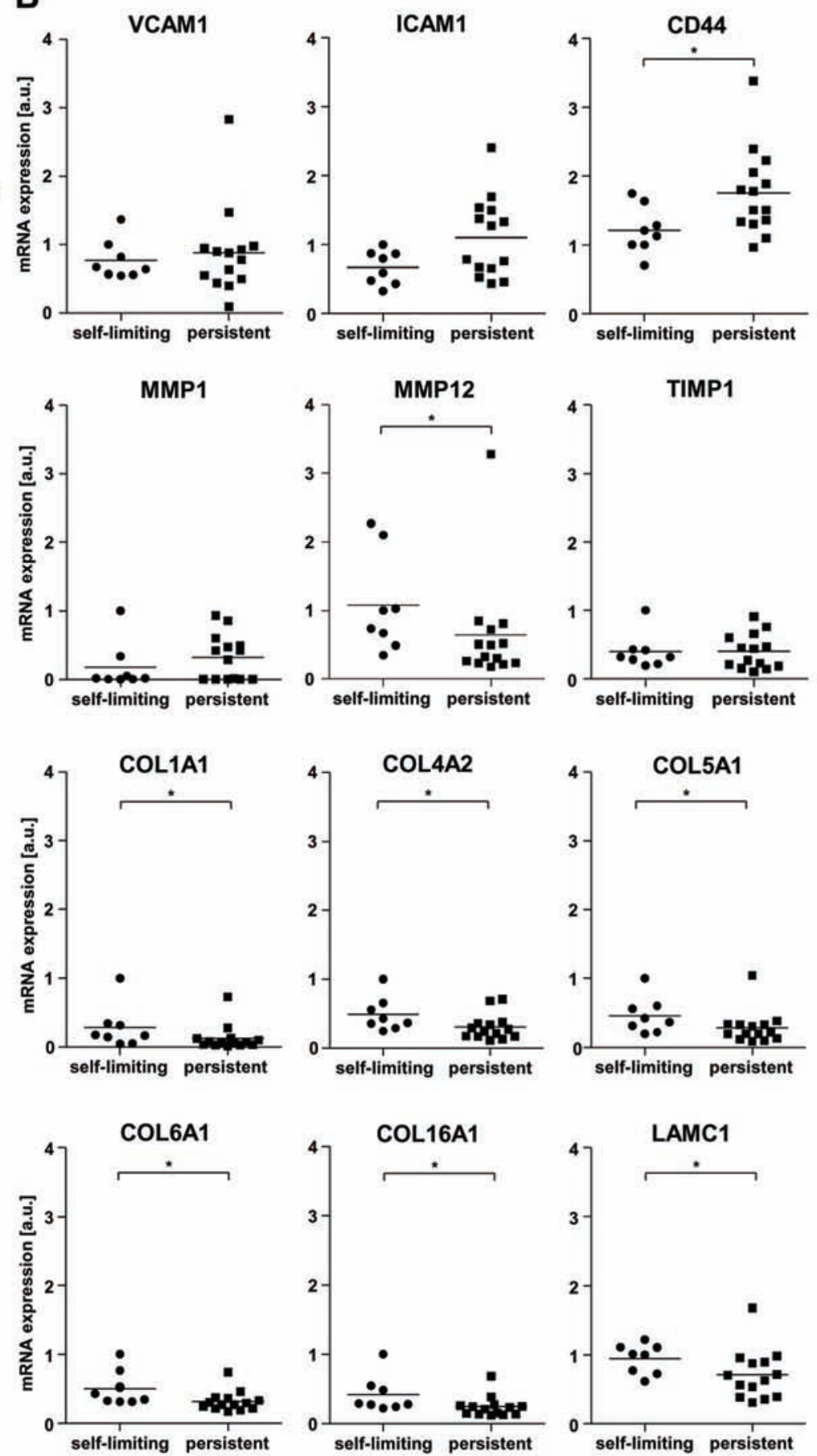

Figure 3 Gene expression analysis in synovial tissue of patients with early rheumatoid arthritis (RA) with self-limiting and persistent disease. A. Supervised hierarchical cluster analysis of expression of 83 genes involved in extracellular matrix (ECM) biology and cellular adhesion. Analysis was performed on patients with $R A$, undifferentiated arthritis $(U A)>>U A$ and $U A>>R A$, pooled and grouped based on self-limiting $(n=8)$ and persistent $(n=14)$ disease. Each column represents the data of one patient and each row shows the relative expression of a given gene for all patients. Gene designations are listed at the right of the figure. Red signifies relatively higher expression of a given gene, green relatively lower expression and black signifies equivalence to the median expression of that gene across all patients. B. Relative mRNA expression of selected genes in patients with selflimiting and persistent disease, expressed as the ratio between the gene of interest and ribosomal protein L13a (RPL13A). Each data point represents a value for an individual patient and bars represent mean values. ${ }^{*} p<0.05$.

Relative JNK activation and is enhanced in patients with persistent disease

Among patients who fulfilled 2010 ACR/EULAR criteria for RA after 2 years of follow-up, 17 patients had persistent non-erosive disease, 12 had erosive disease and 10 had self-limiting disease (supplementary table 4). ERK activation (figure 2C) was elevated in patients with erosive disease compared to those with selflimiting disease $(p<0.005)$, and a trend towards enhanced ERK activation was observed in patients with erosive disease compared to those with persistent non-erosive disease $(p=0.051)$. 
Table 3 Characteristics of patients with early arthritis with erosive and non-erosive disease

\begin{tabular}{lccl}
\hline & Erosive, $\mathbf{n}=\mathbf{1 7}$ & Non-erosive, $\mathbf{n = 3 3}$ & $\mathbf{p ~ V a l u e}$ \\
\hline Age, years & $54(24-81)$ & $46(20-75)$ & 0.92 \\
Disease duration, months & $6(1-11)$ & $5(1-12)$ & 0.39 \\
DAS28 & $3.7(1.1-5.7)$ & $2.4(0.6-6.3)$ & 0.012 \\
ESR, $\mathrm{mm} / \mathrm{h}$ & $36(3-91)$ & $32(9-93)$ & 0.72 \\
CRP, $\mathrm{mg} /$ /itre & $14(1-114)$ & $10(1-133)$ & 0.68 \\
VAS, 0-100 & $42(11-81)$ & $38(3-98)$ & 0.88 \\
IgM-RF positive, \% & 35 & 21 & 0.32 \\
ACPA positive, \% & 24 & 27 & 0.88 \\
\hline
\end{tabular}

ACPA, anti-citrullinated protein antibody; CRP, C-reactive protein; DAS28, 28-joint Disease Activity Score; ESR, erythrocyte sedimentation rate; IgM-RF, immunoglobulin M rheumatoid factor; VAS, visual analogue scale of global disease activity.

JNK activation was significantly lower in those patients with RA with self-limiting disease compared to those with persistent $(p<0.005)$ and erosive disease $(p<0.01)$, associated with lower scores for tender joint count, swollen joint count and DAS28 (supplementary table 4).

As JNK activation was elevated in persistent disease, and often associated with the regulation of expression extracellular matrix (ECM) component biology and cellular interactions with the ECM, we examined expression of a targeted gene set in patients with RA from this cohort with self-limiting $(n=8)$ and persistent (non-erosive and erosive, $\mathrm{n}=14$ ) disease from which mRNA was available. Quantitative PCR (qPCR) analysis was performed on approximately 80 genes encoding ECM components, matrix metalloproteinases (MMPs) and adhesion molecules. Unsupervised hierarchical cluster analysis of gene products failed to group patients according to disease outcome (data not shown), and no trends toward similar gene expression patterns were observed using supervised hierarchical clustering (figure 3A). However, analysis of expression of each individual gene (figure $3 \mathrm{~B}$ and supplementary table 5 ) revealed that expression of collagens COL1A1, COL4A2, COL5A1, COL6A1 and COL16A1, the laminin LAMC1 and MMP-12 were significantly downregulated in patients with persistent disease, while expression of CD 44 was elevated (all $p<0.05$ ).

\section{DISCUSSION}

Because p38, ERK and JNK are each detected in their activated form in the synovial tissue of patients with various forms of inflammatory arthritis, and are requisite for pathology in animal models of arthritis, MAPKs might represent attractive therapeutic targets in the treatment of RA and other forms of arthritis. ${ }^{1-4}$ However, the clinical inefficacy of p38 inhibitors in early trials with patients with RA has indicated a need for a greater understanding of the contributions of MAPKs to synovitis and joint destruction. ${ }^{22} 2337$ Intriguingly, recent kinetic analyses of MAPK activation in multiple murine models of RA have also indicated model-specific differential involvement of each MAPK in distinct phases of initiation, perpetuation and resolution of disease. ${ }^{24}$ This latter observation prompted us to examine MAPK expression and phosphorylation in the earliest stages of human arthritis.

We find that synovial activation of ERK and JNK, but not p38, is significantly elevated in patients with RA compared to patients with other diagnoses, as well as in patients who develop erosive RA. Perhaps important to the aetiology of RA, JNK activation is elevated in patients with RA even before 1987 ACR classification criteria of RA are met. Assessing all patients with early arthritis, regardless of disease diagnosis, we found that each MAPK was more highly activated in patients with erosive disease, but here, only JNK activation predicted the development of erosive disease. Forward and backward linear regression failed to identify roles for p38 and ERK in diagnosis and disease progression (data not shown). Further studies in larger independent cohorts will obviously be needed to assess the positive predictive value and the negative predictive value of JNK activation in predicting RA and erosive disease.

We found no evidence supporting a role for p38 in the onset of RA or eventual joint destruction in arthritis. Phosphorylated p38 is readily detected in the synovial tissue of patients with active and end-stage destructive RA, and mice in the human TNFtransgenic model of RA. ${ }^{3} 421$ In these mice, pharmacological p38 inhibition blocks disease onset. ${ }^{15}$ However, p38 activation in murine collagen-induced arthritis is only modestly increased over baseline until late in disease (day 40-50), when clinical parameters and cytokine biomarkers of disease activity recede. ${ }^{24}$ Also, p38 activity is not affected in TNF-transgenic mice following anti-TNF treatment, or in patients with psoriatic arthritis treated with etanercept. ${ }^{21} 38$ Thus, p38 may contribute significantly to negative feedback mechanisms dampening inflammatory responses or repairing tissue in established RA. ${ }^{39}$ In patients with early arthritis p38-dependent repair mechanisms may not yet have been initiated.

ERK phosphorylation is also elevated in patients with early arthritis diagnosed as having RA and in patients with RA who develop erosive disease. ERK phosphorylation is also readily detected in the synovial tissue of patients with RA undergoing joint replacement. ${ }^{4}$ The extent and timing of ERK activation in experimental arthritis is heavily model-dependent, although genetic or pharmacological suppression of ERK appears to uniformly suppress disease. ${ }^{101724}$ However, as in vitro studies in RA FLS have revealed that many secreted products routinely assessed as TNF-dependent activation markers relevant to RA are relatively insensitive to ERK inhibition. Thus, the mechanisms by which ERK contributes to inflammation and disease progression in the earliest stages of RA remain to be established. ${ }^{9}$

Strikingly, JNK phosphorylation is significantly elevated in synovial tissue from patients with early RA, even before 1987 ACR classification criteria are met. During the course of our study, new classification criteria for the diagnosis of RA were defined, ${ }^{27} 28$ and re-examining our cohort with these criteria, we found that half of the patients originally classified as having UA at baseline now met criteria for RA, similar to a recent study ${ }^{40}$ Importantly, using the new criteria, we confirmed that relative JNK activity is enhanced in early RA. However, insufficient numbers of patients could be classified as UA $>>$ UA to 
allow logistic regression analysis to determine whether JNK activity at baseline was predictive of fulfilment of RA according to the $2010 \mathrm{ACR} / \mathrm{EULAR}$ criteria after follow-up. We also confirmed that ERK activation is enhanced in patients with RA who develop erosive disease. Finally, with the new criteria we identified a subset of patients that fulfil 2010 ACR/EULAR criteria for RA at baseline, but have low levels of JNK activity and self-limiting disease.

Synovial JNK phosphorylation has previously been detected in patients with longstanding RA, and JNK contributes to interleukin 1 13 -induced collagenase expression, inflammation and joint destruction in murine arthritis models. ${ }^{12} 1841$ Additionally, JNK signalling drives collagenase-3 expression and bone damage in rat adjuvant-induced arthritis. ${ }^{12}$ These studies have indicated a role for JNK in regulating synovial ECM biology, or cell interactions with the ECM. Comparing patients with early arthritis with self-limiting disease versus those with persistent disease, either non-erosive or erosive, we find that enhanced JNK activation is associated with decreased expression of developmentally important ECM components, including several forms of collagen, laminin C1 and MMP-12. We also find that CD44 expression is elevated in patients with early arthritis who develop persistent disease. CD44 is thought to have an important role in the pathology of RA, acting as an adhesion molecule and ECM component ligand for endothelial cells, macrophages, lymphocytes and FLS. ${ }^{42}$ Specific splice variants of CD44 are associated with invasive FLS behaviour in RA. ${ }^{43-46}$ Also, a pathogenic role for $\mathrm{CD} 44$ has been demonstrated in animal arthritis models. ${ }^{42} 47$ Further studies will determine if there is a direct relationship between expression of these genes and JNK activity in patients with early arthritis, and if so, whether JNK activation regulates or responds to these genes. Our studies indicate that evaluation of JNK activity might be of additional diagnostic and prognostic value to the new classification criteria, and that pharmacological targeting of JNK and ERK may be of benefit in limiting inflammation and joint destruction early in the development of RA.

Acknowledgements The authors would like to thank Ms Beatrice M Fernandez, Ms LM Hartkamp and Ms IE van Es (University of Amsterdam) for assistance in mRNA preparation, qPCR array gene analysis and immunohistochemical studies.

Funding This research was supported by the Dutch Arthritis Association (NR 04-1-301). Funding was also provided by the European Community's FP6 funding (Autocure). This publication reflects only the views of the authors and the European Community is not liable for any use that may be made of the information herein.

Provenance and peer review Not commissioned; externally peer reviewed.

\section{REFERENCES}

1. Thalhamer $\mathbf{T}$, McGrath MA, Harnett MM. MAPKs and their relevance to arthritis and inflammation. Rheumatology (Oxford) 2008;47:409-14.

2. Schett G, Zwerina J, Firestein G. The p38 mitogen-activated protein kinase (MAPK) pathway in rheumatoid arthritis. Ann Rheum Dis 2008;67:909-16.

3. Han Z, Boyle DL, Aupperle KR, et al. Jun N-terminal kinase in rheumatoid arthritis. J Pharmacol Exp Ther 1999;291:124-30.

4. Schett G, Tohidast-Akrad M, Smolen JS, et al. Activation, differential localization, and regulation of the stress-activated protein kinases, extracellular signalregulated kinase, c-JUN N-terminal kinase, and p38 mitogen-activated protein kinase, in synovial tissue and cells in rheumatoid arthritis. Arthritis Rheum 2000; 43:2501-12

5. Korb A, Tohidast-Akrad M, Cetin E, et al. Differential tissue expression and activation of p38 MAPK alpha, beta, gamma, and delta isoforms in rheumatoid arthritis. Arthritis Rheum 2006;54:2745-56

6. Nishikawa M, Myoui A, Tomita T, et al. Prevention of the onset and progression of collagen-induced arthritis in rats by the potent p38 mitogen-activated protein kinase inhibitor FR167653. Arthritis Rheum 2003;48:2670-81.

7. Westra J, Limburg PC, de Boer P, et al. Effects of RWJ 67657, a p38 mitogen activated protein kinase (MAPK) inhibitor, on the production of inflammatory mediators by rheumatoid synovial fibroblasts. Ann Rheum Dis 2004;63:1453-9.
8. Mbalaviele G, Anderson G, Jones A, et al. Inhibition of p38 mitogen-activated protein kinase prevents inflammatory bone destruction. J Pharmacol Exp Ther 2006;317:1044-53.

9. Kunisch E, Gandesiri M, Fuhrmann R, et al. Predominant activation of MAP kinases and pro-destructive/pro-inflammatory features by TNF alpha in early-passage synovial fibroblasts via TNF receptor-1: failure of $p 38$ inhibition to suppress matrix metalloproteinase-1 in rheumatoid arthritis. Ann Rheum Dis 2007;66:1043-51.

10. Thiel MJ, Schaefer CJ, Lesch ME, et al. Central role of the MEK/ERK MAP kinase pathway in a mouse model of rheumatoid arthritis: potential proinflammatory mechanisms. Arthritis Rheum 2007;56:3347-57.

11. Rockel JS, Bernier SM, Leask A. Egr-1 inhibits the expression of extracellular matrix genes in chondrocytes by TNFalpha-induced MEK/ERK signalling. Arthritis Res Ther 2009;11:R8.

12. Han Z, Boyle DL, Chang L, et al. c-Jun N-terminal kinase is required for metalloproteinase expression and joint destruction in inflammatory arthritis. J Clin Invest 2001;108:73-81.

13. Hammaker DR, Boyle DL, Chabaud-Riou M, et al. Regulation of c-Jun N-terminal kinase by MEKK-2 and mitogen-activated protein kinase kinase kinases in rheumatoid arthritis. J Immunol 2004;172:1612-18.

14. Medicherla S, Ma JY, Mangadu R, et al. A selective p38 alpha mitogen-activated protein kinase inhibitor reverses cartilage and bone destruction in mice with collageninduced arthritis. J Pharmacol Exp Ther 2006;318:132-41.

15. Zwerina $\mathbf{J}$, Hayer $\mathrm{S}$, Redlich $\mathrm{K}$, et al. Activation of $\mathrm{p} 38$ MAPK is a key step in tumor necrosis factor-mediated inflammatory bone destruction. Arthritis Rheum 2006;54:463-72.

16. Hill RJ, Dabbagh K, Phippard D, et al. Pamapimod, a novel p38 mitogen-activated protein kinase inhibitor: preclinical analysis of efficacy and selectivity. J Pharmacol Exp Ther 2008;327:610-19.

17. Fusello AM, Mandik-Nayak L, Shih F, et al. The MAPK scaffold kinase suppressor of Ras is involved in ERK activation by stress and proinflammatory cytokines and induction of arthritis. J Immunol 2006;177:6152-8.

18. Han Z, Chang L, Yamanishi Y, et al. Joint damage and inflammation in C-Jun $\mathrm{N}$-terminal kinase 2 knockout mice with passive murine collagen-induced arthritis. Arthritis Rheum 2002;46:818-23.

19. Sweeney SE, Firestein GS. Mitogen activated protein kinase inhibitors: where are we now and where are we going? Ann Rheum Dis 2006;65 Suppl 3:iii83-8.

20. Tas SW, Remans PH, Reedquist KA, et al. Signal transduction pathways and transcription factors as therapeutic targets in inflammatory disease: towards innovative antirheumatic therapy. Curr Pharm Des 2005;11:581-611.

21. Görtz B, Hayer $\mathrm{S}$, Tuerck B, et al. Tumour necrosis factor activates the mitogenactivated protein kinases p38alpha and ERK in the synovial membrane in vivo. Arthritis Res Ther 2005; 7:R1140-7.

22. Cohen SB, Cheng TT, Chindalore V, et al. Evaluation of the efficacy and safety of pamapimod, a p38 MAP kinase inhibitor, in a double-blind, methotrexate-controlled study of patients with active rheumatoid arthritis. Arthritis Rheum 2009:60:335-44.

23. Damjanov N, Kauffman RS, Spencer-Green GT. Efficacy, pharmacodynamics, and safety of VX-702, a novel p38 MAPK inhibitor, in rheumatoid arthritis: results of two randomized, double-blind, placebo-controlled clinical studies. Arthritis Rheum 2009;60:1232-41.

24. Fukushima A, Boyle DL, Corr M, et al. Kinetic analysis of synovial signalling and gene expression in animal models of arthritis. Ann Rheum Dis 2010;69:918-23.

25. Arnett FC, Edworthy SM, Bloch DA, et al. The American Rheumatism Association 1987 revised criteria for the classification of rheumatoid arthritis. Arthritis Rheum 1988;31:315-24.

26. Dougados M van der Linden S, Juhlin R, et al. The European Spondylarthropathy Study Group preliminary criteria for the classification of spondylarthropathy. Arthritis Rheum 1991;34:1218-27.

27. Aletaha D, Neogi T, Silman AJ, et al. 2010 Rheumatoid arthritis classification criteria: an American College of Rheumatology/European League Against Rheumatism collaborative initiative. Arthritis Rheum 2010;62:2569-81.

28. Aletaha D, Neogi T, Silman AJ, et al. 2010 rheumatoid arthritis classification criteria: an American College of Rheumatology/European League Against Rheumatism collaborative initiative. Ann Rheum Dis 2010;69:1580-8.

29. Smolen JS, Landewé R, Breedveld FC, et al. EULAR recommendations for the management of rheumatoid arthritis with synthetic and biological disease-modifying antirheumatic drugs. Ann Rheum Dis 2010;69:964-75.

30. van der Heijde D, Dankert T, Nieman F, et al. Reliability and sensitivity to change of a simplification of the Sharp/van der Heijde radiological assessment in rheumatoid arthritis. Rheumatology (Oxford) 1999;38:941-7.

31. Gerlag DM, Tak PP. How to perform and analyse synovial biopsies. Best Pract Res Clin Rheumatol 2009;23:221-32.

32. Gerlag D, Tak PP. Synovial biopsy. Best Pract Res Clin Rheumatol 2005;19:387-400.

33. Smeets TJ, Barg EC, Kraan MC, et al. Analysis of the cell infiltrate and expression of proinflammatory cytokines and matrix metalloproteinases in arthroscopic synovial biopsies: comparison with synovial samples from patients with end stage destructive rheumatoid arthritis. Ann Rheum Dis 2003;62:635-8.

34. Haringman JJ, Vinkenoog M, Gerlag DM, et al. Reliability of computerized image analysis for the evaluation of serial synovial biopsies in randomized controlled trials in rheumatoid arthritis. Arthritis Res Ther 2005;7:R862-7. 
35. Rantapää-Dahlqvist S, de Jong BA, Berglin E, et al. Antibodies against cyclic citrullinated peptide and IgA rheumatoid factor predict the development of rheumatoid arthritis. Arthritis Rheum 2003;48:2741-9.

36. Berglin E, Johansson T, Sundin U, et al. Radiological outcome in rheumatoid arthritis is predicted by presence of antibodies against cyclic citrullinated peptide before and at disease onset, and by IgA-RF at disease onset. Ann Rheum Dis 2006;65:453-8.

37. Genovese MC. Inhibition of p38: has the fat lady sung? Arthritis Rheum 2009;60:317-20.

38. Lories RJ, Derese I, Luyten FP, et al. Activation of nuclear factor kappa B and mitogen activated protein kinases in psoriatic arthritis before and after etanercept treatment. Clin Exp Rheumatol 2008;26:96-102.

39. Clark AR, Dean JL, Saklatvala J. The p38 MAPK pathway mediates both antiinflammatory and proinflammatory processes: comment on the article by Damianov and the editorial by Genovese. Arthritis Rheum 2009:60:3513-14

40. van der Linden MP, Knevel R, Huizinga TW, et al. Classification of rheumatoid arthritis: comparison of the 1987 American College of Rheumatology criteria and the 2010 American College of Rheumatology/European League Against Rheumatism criteria. Arthritis Rheum 2011:63:37-42.
41. Guma M, Kashiwakura J, Crain B, et al. JNK1 controls mast cell degranulation and IL-1 \{beta\} production in inflammatory arthritis. Proc Natl Acad Sci USA 2010;107:22122-7.

42. Naor D, Nedvetzki S. CD44 in rheumatoid arthritis. Arthritis Res Ther 2003;5:105-15.

43. Wibulswas A, Croft D, Bacarese-Hamilton I, et al. The CD44v7/8 epitope as a target to restrain proliferation of fibroblast-like synoviocytes in rheumatoid arthritis. Am J Pathol 2000;157:2037-44.

44. Wibulswas A, Croft D, Pitsillides AA, et al. Influence of epitopes CD44v3 and CD44v6 in the invasive behavior of fibroblast-like synoviocytes derived from rheumatoid arthritic joints. Arthritis Rheum 2002;46:2059-64.

45. Tolboom TC, Huidekoper AL, Kramer IM, et al. Correlation between expression of CD44 splice variant v8-v9 and invasiveness of fibroblast-like synoviocytes in an in vitro system. Clin Exp Rheumatol 2004;22:158-64.

46. Neidhart M, Gay RE, Gay S. Anti-interleukin-1 and anti-CD44 interventions producing significant inhibition of cartilage destruction in an in vitro model of cartilage invasion by rheumatoid arthritis synovial fibroblasts. Arthritis Rheum 2000;43:1719-28.

47. Mikecz K, Dennis K, Shi M, et al. Modulation of hyaluronan receptor (CD44) function in vivo in a murine model of rheumatoid arthritis. Arthritis Rheum 1999:42:659-68. 